MAdROÑO, Vol. 59, No. 4, p. 236, 2012

\title{
REVIEWERS OF MADROÑO MANUSCRIPTS 2012
}

\author{
Paul Beardsley \\ Elizabeth Braker \\ Leo Bruederle \\ James Cohen \\ Tom Daniel \\ Naomi Fraga \\ Timothy Griffith \\ Lisa Grubisha \\ Richard Halse \\ Dylan Hannon \\ Chris Havran \\ Christina Hazard \\ Kathleen Kay \\ Jon Keely \\ Ron Kelley \\ Byron Lamont \\ Thomas Marcussen
}

Bruce Maslin

Stephen McCabe

Nicole Molinari

Nancy Morin

Tom Parker

Robert Patterson

Jon Rebman

Michael Simpson

Shannon Still

Dean Taylor

Mike Vasey

Michael Vincent

Mark Waldrop

Gary Wallace

Dieter Wilken

Nina Wurzburger

Jenn Yost 


\section{$2 \mathrm{BHL}$ Biodiversity Heritage Library}

2012. "Reviewers of Madroño Manuscripts 2012." Madroño; a West American journal of botany 59, 236-236. https://doi.org/10.3120/0024-9637-59.4.236.

View This Item Online: https://www.biodiversitylibrary.org/item/185599

DOI: https://doi.org/10.3120/0024-9637-59.4.236

Permalink: https://www.biodiversitylibrary.org/partpdf/169177

\section{Holding Institution}

Smithsonian Libraries

\section{Sponsored by}

Biodiversity Heritage Library

\section{Copyright \& Reuse}

Copyright Status: In Copyright. Digitized with the permission of the rights holder License: http://creativecommons.org/licenses/by-nc/3.0/ Rights: https://www.biodiversitylibrary.org/permissions/

This document was created from content at the Biodiversity Heritage Library, the world's largest open access digital library for biodiversity literature and archives. Visit BHL at https://www.biodiversitylibrary.org. 